Pacific Journal of Mathematics

QUASIREGULAR NEARNESS SPACES AND EXTENSIONS OF

MAY AND OLAV NJÈ\&STAD 


\title{
QUASI-REGULAR NEARNESS SPACES AND EXTENSIONS OF NEARNESS-PRESERVING MAPS
}

\author{
K. C. Chattopadhyay and Olav NuÅstad
}

\begin{abstract}
Every basic nearness (or quasi-nearness) induces a Čech closure operator. There is a 1-1 correspondence between the cluster generated Riesz nearnesses on a given $T_{1}$ closure space and the principal (or strict) $T_{1}$ extensions of the space. (In particular linkage compact extensions correspond to proximal nearnesses, $F$-linkage compact extensions correspond to contigual nearnesses, while ordinary compact extensions correspond to cluster generated weakly contigual nearnesses.

In this paper we discuss conditions under which a nearness-preserving map between nearness spaces can be extended to a continuous map between the corresponding principal extensions of the induced closure spaces. The concept of a quasi-regular nearness space plays an important role in this connection. The general results on extensions of nearnesspreserving maps are used to obtain results on extension of continuous maps into regular linkage compact and $F$-linkage compact spaces.
\end{abstract}

1. Introduction. Nearnesses on a topological space can be used as a means of introducing extensions of the space. Work of Bentley [1], Bentley and Herrlich [2], Herrlich [8], Naimpally [11] and Reed [12] show that every Lodato nearness on a given $T_{1}$ topological space gives rise to a principal (or strict) $T_{1}$ extension of the space, and all the principal $T_{1}$ extensions can be obtained in this way. (Compact extensions correspond to contigual nearnesses and linkage compact (or clan complete) extensions correspond to proximal nearnesses.) A nearness-preserving map between nearness spaces can be extended to a continuous map between the corresponding extensions of the underlying topological space, if suitable regularity conditions are imposed on the image space.

Basic nearnesses (quasi-nearnesses in the terminology of Herrlich [8]) induce closure spaces rather than topological spaces. It was recently shown by Chattopadhyay, Njåstad and Thron [4] that the above correspondence between Lodato nearnesses and principal extensions can be extended: Every Riesz nearness on a given $T_{1}$ closure space gives rise to a principal $T_{1}$ extension of the space, and all the principal $T_{1}$ extensions can be obtained in this way. (Linkage compact extensions correspond to proximal nearnesses, $F$-linkage compact extensions correspond to contigual nearnesses, while compact extensions correspond to weakly contigual cluster generated nearnesses.)

In the present paper we discuss conditions under which a nearnesspreserving map between (basic) nearness spaces can be extended to a continuous map between the corresponding principal extensions of the 
induced closure spaces. The concept of a quasi-regular nearness space, which generalizes the concept of a regular nearness space, plays an important role. Our results include earlier known results for the case that the induced closure spaces are topological.

The general results on extension are used to obtain results on extension of continuous maps into regular linkage compact and $F$-linkage compact spaces. These results are given a categorical formulation.

\section{Preliminaries.}

2.1. A closure operator $c$ on a set $X$ is a function from $\mathscr{P} X$ into $\mathscr{P} X$ satisfying the following conditions:

$$
\begin{aligned}
& \mathrm{C} 1: c \varnothing=\varnothing, \\
& \mathrm{C} 2: c A \supset A, \\
& \mathrm{C} 3: c(A \cup B)=c A \cup c B .
\end{aligned}
$$

A pair $(X, c)$, where $c$ is a closure operator on $X$, is called a closure space. Closure spaces are studied in detail in Čech [5]. (A topological space is a closure space where the closure operator in addition satisfies $c c A=c A$.)

The interior of a set $B$ is defined by $i B=X-c(X-B)$. The set $V$ is called a neighborhood of the point $x$ if $x \in i V$. All the neighbourhoods of $x$ form a filter $\mathcal{F}(c, x)$, the neighbourhood filter of $x$.

The closure space $(X, c)$ is said to be a $T_{1}$ space if $c\{x\}=\{x\}$ for every $x \in X$, a $T_{2}$ space if any two distinct points have disjoint neighbourhoods. For topological spaces these definitions clearly coincide with the usual ones.

A grill $\mathcal{G}$ on $X$ is a family of subsets satisfying

$$
\varnothing \notin \mathcal{G}, \quad B \supset A \in \mathcal{G} \Rightarrow B \in \mathcal{G}, \quad A \cup B \in \mathcal{G} \Rightarrow A \in \mathcal{G} \text { or } \quad B \in \mathcal{G} \text {. }
$$

For every family $C$ of subsets of $X$ let sec $\bigodot$ denote the family $\{D \subset X: D \cap C \neq \varnothing$ for all $C \in \mathcal{C}\}$. The function $\mathcal{C} \rightarrow$ sec $\mathcal{C}$ establishes a 1-1 correspondence between the set of filters on $X$ and the set of grills on $X$ : If $\mathscr{F}$ is a filter then $\sec \mathscr{F}$ is a grill, if $\mathcal{G}$ is a grill then $\sec \mathcal{G}$ is a filter, and $\sec ^{2} \mathscr{F}=\mathscr{F}, \sec ^{2} \mathcal{G}=\mathcal{G}$. For every point $x$ of the closure space $(X, c)$ the family $\mathcal{G}(c, x)=\sec \mathscr{F}(c, x)=\{A: x \in c A\}$ is a grill, and is called the adherence grill of $x$.

2.2. A grill $\mathcal{G}$ is said to be $F$-linked if $\cap_{k=1}^{n} c A_{k} \neq \varnothing$ for every finite subfamily $\left\{A_{1}, \ldots, A_{n}\right\}$ of $\mathcal{G}$. It is said to be linked if $c A \cap c B \neq \varnothing$ for every two elements $A, B$ of $\mathcal{G}$. The space $(X, c)$ is said to be $F$-linkage compact if every $F$-linked grill is contained in some adherence grill $\mathcal{S}(c, x)$, linkage compact if every linked grill is contained in some $\mathcal{G}(c, x)$. For more details see Chattopadhyay, Njåstad and Thron [4]. F-linkage compactness coincides with compactness when $(X, c)$ is a topological space. 
Linkage compact topological spaces were considered by Reed [12] (she called them clan complete spaces). Linkage compactness coincides with compactness when $(X, c)$ is a topological $T_{2}$ space.

2.3. A collection $\nu \subset \mathscr{P}^{2} X$ is called a nearness (or a basic nearness) on $X$ if it satisfies the following conditions:

$\mathrm{N} 1: \mathbb{Q}<\mathscr{B} \in \nu \Rightarrow \mathbb{Q} \in \nu$,

N2: $\cap\{A: A \in \mathbb{Q}\} \neq \varnothing \Rightarrow \mathbb{Q} \in \nu$,

$\mathrm{N} 3: \mathbb{Q} \in \nu \Rightarrow \varnothing \notin \mathbb{Q}$,

N4: $\mathbb{Q} \vee \mathscr{B} \in \nu \Rightarrow \mathbb{Q} \in \nu$ or $\mathscr{B} \in \nu$.

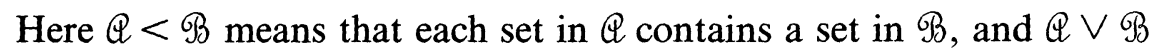
denotes the family $\{A \cup B: A \in \mathbb{Q}, B \in \Re\}$.

A pair $(X, \nu)$, where $\nu$ is a (basic) nearness, is called a nearness space. Every (basic) nearness $\nu$ on $X$ induces a closure operator $c_{\nu}$ as follows:

$$
c_{\nu} A=\{x:\{\{x\}, A\} \in \nu\} .
$$

A nearness $\nu$ which in this way induces a closure operator $c$ (i.e. for which $c_{\nu}=c$ ) is said to be compatible with $c$.

We shall call $\nu$ a $T_{1}$ nearness if $\left(X, c_{\nu}\right)$ is a $T_{1}$ space, a $T_{2}$ nearness if $\left(X, c_{\nu}\right)$ is a $T_{2}$ space.

What we have called a (basic) nearness is called a quasi-nearness by Herrlich [8]. A collection which in addition to N1-N4 also satisfies the condition

$$
\text { N5: }\left\{c_{\nu} A: A \in \mathbb{Q}\right\} \in \nu \Rightarrow \mathbb{Q} \in \nu
$$

is called a nearness by Herrlich [8]. We find it convenient in this paper to use the term nearness for collections satisfying N1-N4, and the term Lodato nearness for collections satisfying N1-N5 (cf. e.g. Reed [12]).

Structures equivalent to (basic) nearnesses, but formulated in terms of micromeric families (that is in terms of the families $\sec Q, Q \in \nu$ ) were studied by Katětov [9] under name merotopic structures, see also Herrlich [8, p. 76].

A nearness $\nu$ which in addition to N1-N4 satisfies the condition

N6: $\left\{c_{\nu} A: A \in \mathbb{Q}\right\} \neq \varnothing \Rightarrow \mathbb{Q} \in \nu$ we shall call a Riesz nearness. The condition N6 is clearly weaker than the condition N5.

A Lodato nearness always induces a topological closure space. The closure space $\left(X, c_{\nu}\right)$ may be topological also if the space $(X, \nu)$ is not a Lodato space.

A grill belonging to $\nu$ is called a $(\nu$-)clan. A maximal family in $\nu$ is called a $(\nu$-)-cluster. Every $\nu$-cluster is a $\nu$-clan. A nearness $\nu$ is easily seen to be a Riesz nearness iff for all $x \in X$ the adherence grills $\mathcal{G}\left(c_{\nu}, x\right)$ are $\nu$-clusters. Clusters of the form $\mathcal{G}\left(c_{\nu}, x\right)$ are called principal clusters. 
2.4. A nearness $\xi$ is called a contigual nearness if it satisfies the condition

$Q \in \xi$ iff every finite subfamily of $Q$ belongs to $\xi$.

A nearness $\pi$ is called a proximal nearness if it satisfies the condition

$Q \in \pi$ iff $Q$ is contained in a grill $\mathcal{G}$ every two-set subfamily of which belongs to $\pi$.

Every proximal nearness is contigual.

For every $T_{1}$ closure space $(X, c)$ there exists at least one compatible nearness $\nu$. In particular there is a smallest (in the set theoretic sense) Riesz nearness $\nu(c)$, a smallest contigual Riesz nearness $\xi(c)$ and a smallest proximal Riesz nearness $\pi(c)$ compatible with $c$. These nearnesses are defined as follows:

$$
\begin{aligned}
& \mathbb{Q} \in \nu(c) \text { iff } \cap\{c A: A \in \mathbb{Q}\} \neq \varnothing, \\
& \mathbb{Q} \in \xi(c) \text { iff } \cap_{k=1}^{n} c A_{k} \neq \varnothing \text { for every finite subfamily }\left\{A_{1} \cdots A_{n}\right\} \\
& \text { of } \mathbb{Q} \text {, }
\end{aligned}
$$

For more detailed information on the concepts discussed in $\$ 2$, see Chattopadhyay and Thron [3], Chattopadhyay, Njåstad and Thron [4], Herrlich [8], Thron [14].

3. Regularity concepts. For nearness-preserving maps to have continuous extensions, some kind of regularity property related to the original regularity concept for topological spaces is needed. In this section we discuss some such concepts for nearness spaces, and their relationship to regularity of the underlying closure spaces.

3.1. Definition. A closure space $(X, c)$ is said to be regular if for each $A \subset X$ and each $x \notin c A$ there exist disjoint neighbourhoods $U$ and $V$ of $x$ and $A$ respectively (i.e.: $U \cap V=\varnothing, x \notin c(X-U), A \cap c(X-V)$ $=\varnothing)$.

This definition was used by Čech [5, p. 492]. If a closure space is regular and topological, then it is clearly a regular topological space in the usual sense.

3.2. ExAMPLE. For further reference we describe this example of a regular, non-topological closure space. (Cf. Čech [5, p. 495].)

Set $X_{n}=\{(1,1 / n),(2,1 / n), \ldots,(m, 1 / n), \ldots\}$, for $n=1,2, \ldots, J=$ $\{1,2, \ldots, n, \ldots\}, Y=\cup_{i=1}^{\infty} X_{i}, Z=J \cup\{\omega\}, X=Y \cup Z$. (Here $\omega$ is an element not belonging to $Y \cup J$.) 
We define

$c_{1} A=A \cup\left\{n \in J: A \cap X_{n}\right.$ is infinite $\}$ if $A$ is a subset of $Y$, $c_{2} A=A$ if $A$ is a finite subset of $Z$, $c_{2} A=A \cup\{\omega\} \quad$ if $A$ is an infinite subset of $Z$.

Finally for each subset $A$ of $X$ we define

$$
c A=c_{1}(A \cap Y) \cup c_{2}(A \cap Z) .
$$

Clearly $c$ is a closure operator on $X$, and the closure space $(X, c)$ is a $T_{1}$ space. Note that $c Y=c_{1} Y=Y \cup J$ and $c c Y=c_{1} Y \cup c_{2} J=X$. Hence $c Y \neq c c Y$, and thus $(X, c)$ is not a topological space.

Now suppose that $A \subset X, x \notin c A$.

First assume that $x \in Y$. Set $U=\{x\}, V=X-\{x\}$. Then clearly $c(X-U)=c V=V, c(X-V)=c U=\{x\}$.

Next assume that $x=n \in J$. Set $U=\left(X_{n}-A\right) \cup\{n\}, V=X-U$ $=\left(X_{n} \cap A\right) \cup\left(\cup\left\{X_{k}: k \neq n\right\}\right) \cup(Z-\{n\})$. Clearly $c U=U$, and hence $A \cap c(X-V)=A \cap c U=A \cap U=\varnothing$. Since $n \notin c A$ the set $A \cap X_{n}$ is finite, and therefore $c(X-U)=c\left(X_{n} \cap A\right) \cup c\left(\cup\left\{X_{k}: k \neq n\right\}\right) \cup$ $c(Z-\{n\})=\left(X_{n} \cap A\right) \cup\left(\cup\left\{X_{k}: k \neq n\right\}\right) \cup(Z-\{n\})=V$. Thus $x$ $=n \notin c(X-U)$.

Finally assume that $x=\omega$. Set $U=Z-A$ and $V=X-U=Y \cup$ $(Z \cap A)$. Clearly $c U=U$, since $\omega \in U$. Since $\omega \notin c A$ the set $A \cap Z$ is finite, and therefore $c(X-U)=c V=Y \cup J$. Thus $x=\omega \notin c(X-U)$.

So in all three cases, $U$ and $V$ are neighbourhoods of $x$ and $A$ in $(X, c)$, and $U \cap V=\varnothing$.

Hence $(X, c)$ is a regular closure space.

3.3. Definition. A nearness space $(X, \nu)$ is said to be regular if the family $Q$ belongs to $\nu$ whenever the family $\{B \subset X: B$ is a $\nu$-neighbourhood of some $A$ in $\mathbb{Q}$ \} belongs to $\nu$. (A $\nu$-neighbourhood of $A$ is a set $B$ such that $\{A, X-B\} \notin \nu$.)

This definition was introduced by Herrlich [8, p. 88]. It can easily be shown that if $(X, \nu)$ is a regular nearness space, then $(X, \nu)$ is a Lodato nearness space, and $\left(X, c_{\nu}\right)$ is a regular topological space. Structures equivalent to regular nearnesses were studied by Morita [9] and Steiner and Steiner [13].

Every uniform space (given by a collection of uniform covers) determines a nearness space $(X, \nu)$, where a family $Q$ belongs to $\nu$ iff the family $\{X-A: A \in \mathbb{Q}\}$ is not a uniform cover. We may thus talk about a uniform nearness space. It is well known that every uniform nearness space is regular. (See Herrlich [8, p. 78-89].) 
3.4. Definition. A nearness space $(X, \nu)$ is said to be an $R$-nearness space if for each $A \subset X$ and $x \notin c_{\nu} A$ there exists a set $V$ such that $\{\{x\}, V\} \notin \nu$ and $[A, X-V] \notin \nu$. The $R$-nearnesses were introduced by Naimpally [11, p. 242], and are modelled after the $R$-proximities of Harris [6]. Actually Naimpally also assumed $(X, \nu)$ to be a $T_{1}$ Lodato nearness space. It is easily seen that if $(X, \nu)$ is an $R$-nearness space, then $\left(X, c_{\nu}\right)$ is a regular topological space, even without assuming the nearness space $(X, \nu)$ to be a Lodato space.

3.5. In the following, a nearness space $(X, \nu)$ is always assumed to be a Riesz nearness space. We define $X^{\nu}$ to be the set of all $\nu$-clusters. For every subset $A$ of $X$ we define $A^{\nu}=\left\{\complement \in X^{\nu}: A \in \mathcal{C}\right\}$. We shall denote by $\Pi$ the set of all principal $\nu$-clusters (that is the set of all $c_{\nu}$-adherence grills). Furthermore $\Omega$ (or $\Omega_{\nu}$ if explicit reference to the nearness $\nu$ is necessary) shall always denote a set of clusters containing $\Pi$, i.e.: $\Pi \subset \Omega \subset X^{\nu}$. For a given $\Omega$ we define $A^{*}=A^{\nu} \cap \Omega$, and $A^{+}=\left\{\mathcal{G}\left(c_{\nu}, a\right): a \in A\right\} \cup$ $\left(A^{*}-\Pi\right)$.

We shall later construct extensions of the closure space $\left(X, c_{\nu}\right)$ where the elements of $\Omega$ represent the points of the extension and where for each element $\mathcal{C}$ of $\Omega$ the family sec $\bigodot$ represents the trace on $X$ of the neighbourhood filter of the element $\mathcal{C}$. We shall introduce a regularity concept on the nearness space $(X, \nu)$ which in a sense is reflected in the regularity property of this closure space extension. Thus this kind of regularity of $(X, \nu)$ will insure that the closure space extension is a regular closure space. (See Proposition 4.2.)

3.6. Definition. A nearness space $(X, \nu)$ is said to be $\Omega$-quasi-regular if for each $\mathcal{C}_{0} \in \Omega$ and each $A \notin \mathcal{C}_{0}$ there exist disjoint subsets $U, V$ of $X$ such that $U \in \sec \mathcal{C}_{0}$ and $V \in \sec \mathcal{C}$ for all $\mathcal{C} \in A^{+}$.

Note that if $\Omega_{1} \subset \Omega_{2}$, then if $(X, \nu)$ is $\Omega_{2}$-regular it is also $\Omega_{1}$-quasiregular. A $X^{\nu}$-quasi-regular space is simply called quasi-regular. Thus if the space $(X, \nu)$ is quasi-regular then it is $\Omega$-quasi-regular for every $\Omega$.

\subsection{Proposition. A regular nearness space is quasi-regular.}

Proof. Let $\bigodot_{0}$ be a cluster and $A_{0}$ a subset of $X$ such that $A_{0} \notin \bigodot_{0}$. Then $\left\{A_{0}\right\} \cup \bigodot_{0} \notin \nu$. Since $\left\{B \subset X: B\right.$ is a $\nu$-neighbourhood of $A_{0}$ or $B$ is a $\nu$-neighbourhood of some $\left.A \in \mathcal{C}_{0}\right\} \notin \nu$ (by definition of regularity), it follows that $\left\{B \subset X: B\right.$ is a $\nu$-neighbourhood of $\left.A_{0}\right\} \not \bigodot_{0}$. Thus there is a $\nu$-neighbourhood $U$ of $A_{0}$ such that $U \notin \bigodot_{0}$. Set $V=X-U$. Then

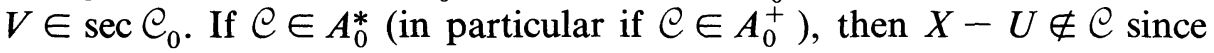
$\left\{A_{0}, X-U\right\} \notin \nu$, and hence $U \in \sec \circlearrowright$. Since $U \cap V=\varnothing$ this shows that $(X, \nu)$ is quasi-regular. 
3.8. Proposition. If $(X, \nu)$ is $\Omega$-quasi-regular then $\left(X, c_{\nu}\right)$ is a regular closure space.

Proof. Let $x \notin c_{\nu} A$. Then $A \notin \mathcal{G}\left(c_{\nu}, x\right) \in \Omega$. Hence there exist disjoint subsets $U, V$ of $X$ such that $U \in \sec \mathcal{G}\left(c_{\nu}, x\right)=\mathscr{F}\left(c_{\nu}, x\right)$ and $V \in$ $\sec \bigodot$ for all $\bigcup \in A^{+}$. Since for each $a \in A, \mathcal{G}\left(c_{\nu}, a\right) \in A^{+}$, it follows that $V \in \sec \mathcal{G}\left(c_{\nu}, a\right)=\mathscr{F}\left(c_{\nu}, a\right)$ for each $a \in A$. Thus $U$ is a neighbourhood of $x$ and $V$ is a neighbourhood of $A$, which shows that $\left(X, c_{\nu}\right)$ is a regular closure space.

3.9. Remark. We may define the nearness space $(X, \nu)$ to be strongly $\Omega$-quasi-regular (strongly quasi-regular) if for every cluster $\bigodot_{0} \in \Omega$ (every cluster $\bigodot_{0} \in X^{\nu}$ ) and every $A \notin \bigodot_{0}$ there exist disjoint subsets $U, V$ of $X$ such that $U \in \sec \bigodot_{0}$ and $V \in \sec \bigodot$ for all $\bigodot \in A^{*}$ (for all $\bigodot \in A^{\nu}$ ). Then the following relationships hold:

(i) From the proof of Proposition 3.7 it immediately follows that a regular nearness space is strongly quasi-regular.

(ii) If $(X, \nu)$ is an $\Omega$-quasi-regular Lodato nearness space, then $(X, \nu)$ is strongly $\Omega$-quasi-regular. (Let $\bigodot_{0} \in \Omega, A \notin \bigodot_{0}$. Then $c_{\nu} A \notin \bigodot_{0}$ since $\bigodot_{0}$ is a cluster in a Lodato nearness space. Hence there exist disjoint sets $U, V$ such that $U \in \sec \bigodot_{0}, V \in \sec \bigodot$ for all $\bigodot \in\left(c_{\nu} A\right)^{+}$, and $a$ fortiori for all $\circlearrowright \in A^{*}$, since clearly $A^{*} \subset\left(c_{\nu} A\right)^{+}$.)

(iii) If $(X, \nu)$ is strongly $\Omega$-quasi-regular, then $\left(X, c_{\nu}\right)$ is a (regular) topological closure space, whether $(X, \nu)$ is a Lodato space or not. (Let $x \notin c_{\nu} A$. Then $A \notin \mathcal{G}\left(c_{\nu}, x\right)$, and so there exist disjoint subsets $U, V$ of $X$ such that $U \in \sec \mathcal{G}\left(c_{\nu}, x\right), V \in \sec \mathcal{C}$ for all $\mathcal{E} \in A^{*}$. In particular $V \in$ $\sec \mathcal{G}\left(c_{\nu}, a\right)$ for all $a \in c_{\nu} A$. Then $c_{\nu} A \cap c_{\nu}(X-U)=\varnothing$ and $x \notin$ $c_{\nu}(X-U)$. It follows that $c_{\nu} A \subset V \subset X-U$ and hence $c_{\nu} c_{\nu} A \subset$ $c_{\nu}(X-U)$. Consequently $x \notin c_{\nu} c_{\nu} A$, which means that $\left(X, c_{\nu}\right)$ is topological.)

We may sum up the situation as follows:

Regular nearness spaces are strongly quasi-regular, and strongly quasi-regular nearness spaces are quasi-regular. Furthermore quasi-regular Lodato nearness spaces are strongly quasi-regular. We have no results relating $R$-nearness spaces to regular, strongly quasi-regular or quasi-regular nearness spaces.

Furthermore quasi-regular nearness spaces induce regular closure operators. Strongly quasi-regular nearness spaces, regular nearness spaces and $R$-nearness spaces all induce regular topological closure operators. Moreover, regular nearness spaces are always Lodato nearness spaces, while this need not be the case for $R$-nearness spaces or strongly quasiregular nearness spaces. 
3.10. Proposition. Let $(X, \nu)$ be a nearness space and assume that $\left(X, c_{\nu}\right)$ is a regular closure space. Then $(X, \nu)$ is $\Pi$-quasi-regular.

Proof. Let $A \notin \mathcal{G}\left(c_{\nu}, x\right)$. Then $x \notin c_{\nu} A$. Since $\left(X, c_{\nu}\right)$ is regular there are disjoint subsets $U, V$ of $X$ such that $x \notin c_{\nu}(X-U)$ and $A \cap$ $c_{\nu}(X-V)=\varnothing$. Clearly $U \in \sec \mathcal{G}\left(c_{\nu}, x\right)$ and $V \in \sec \mathcal{G}\left(c_{\nu}, a\right)$ for all $a \in A$. Since $A^{+}=\left\{\mathcal{G}\left(c_{\nu}, a\right): a \in A\right\}$ when $\Omega=\Pi$ it follows that $(X, \nu)$ is $\Pi$-quasi-regular.

3.11. We recall the definition of the nearness $\nu(c)$ (see $\$ 2.4)$. Note that if $(X, c)$ is a $T_{1}$ space, then $(X, \nu(c))$ is a nearness space such that $c_{\nu(c)}=c$ and $\Pi$ is the set of all $\nu(c)$-clusters. Thus in view of Proposition 3.10 we have the following corollary.

3.12. Corollary. If $(X, c)$ is a regular $T_{1}$ closure space then $(X, \nu(c))$ is a quasi-regular nearness space.

3.13. EXAMPLE. Let $(X, c)$ be the closure space of Example 3.2, and let $Y$ and $J$ have the same meaning as in that example. We shall use this space to construct an example of a quasi-regular nearness which induces a nontopological closure space, and an example of a topological closure space which is induced by a nearness which is not a Lodato nearness.

(i) Let $\nu$ be the nearness $\nu(c)$ on $X$. It follows from Corollary 3.12 that $(X, \nu)$ is a quasi-regular nearness space. Since $c_{\nu(c)}=c$ this gives an example of a quasi-regular nearness inducing a non-topological closure space.

(ii) Let $\mu$ be the restriction of $\nu$ to the subset $Y \cup J=X-\{\omega\}$ of $X$. Note that the nearness $\mu$ induces a topological closure space $\left(Y \cup J, c_{\mu}\right)$. The nearness space $(Y \cup J, \mu)$ is not a Lodato nearness space, however, in view of Remark 3.9. (Or by direct argument: Let the family $\Re$ on $Y \cup J$ be defined by

$B \in \mathscr{B}$ iff $B$ contains the union of an infinite number of the sets $X_{n}$.

Set $B_{1}=\cup_{m=1}^{\infty} X_{2 m}, B_{2}=\cup_{m=0}^{\infty} X_{2 m+1}$. Then clearly $B_{1}, B_{2} \in \mathscr{B}, c B_{1} \cap$ $c B_{2}=\varnothing$, and hence $\mathscr{B} \notin \mu$. On the other hand, $\omega \in c_{\nu} c_{\mu} B$ for every $B \in \Re$, hence $\left\{c_{\mu} B: B \in \Re\right\} \in \mu$. Thus condition N5 is not satisfied). So this gives an example of a topological closure space which is induced by a nearness which is not a Lodato nearness.

4. Principal extensions. When a topological closure space $(X, c)$ is densely embedded in a topological closure space $(Y, k)$, this latter space is said to be a principal (or strict) extension of $(X, c)$ if the closures in $Y$ of the subsets of $X$ form a base for the closed sets in $(Y, k)$. For $T_{1}$ spaces 
the points of $Y$ are in 1-1 correspondence with the trace system on $X$ of $(Y, k)$. (That is: A point $y$ of $Y$ corresponds to the family of sets on $X$ whose closures in $Y$ contain $y$.)

For a non-topological closure space this procedure does not in general lead to an extension of the space. However, by modifying the approach, principal extensions with properties to a certain extent similar to those of principal extensions of topological closure spaces can be obtained. In this section we discuss the construction of such principal extensions when the trace system is a given set $\Omega$ of $\nu$-clusters, where $\nu$ is a nearness compatible with the given closure space.

4.1. Let $(X, \nu)$ be a $T_{1}$ Riesz nearness space. We recall the notations introduced in $\S 3.5$. In particular $\Omega$ shall denote a set of $\nu$-clusters, $\Pi \subset \Omega \subset X^{\nu}$. We define

$$
\begin{aligned}
\psi(x) & =\mathcal{G}\left(c_{\nu}, x\right) \text { for all } x \in X, \\
d \alpha & =\bigcap\left\{A^{*}: A^{*} \supset \alpha\right\} \quad \text { for all } \alpha \subset \Omega, \\
g \alpha & =\left(\psi^{-1}(\alpha)\right)^{*} \cup d(\alpha-\psi(X)) \text { for all } \alpha \subset \Omega .
\end{aligned}
$$

(We write $\psi_{\nu}, d_{\nu}, g_{\nu}$ when it is necessary to refer explicitly to the nearness $\nu$.) The map $\psi$ may be considered as a map from $X$ into $\Omega$ for arbitrary $\Omega$. When $(X, \nu)$ is a $T_{1}$ nearness space, then $(\Omega, g)$ is a $T_{1}$ closure space, $\psi(X)$ is dense in $\Omega$, and $\psi$ is a homeomorphism of $\left(X, c_{\nu}\right)$ onto a subspace of $(\Omega, g)$. (A map $f:\left(X_{1}, c_{1}\right) \rightarrow\left(X_{2}, c_{2}\right)$ is continuous if $f\left(c_{1} A\right) \subset c_{2} f(A)$ for all $A \subset X_{1}$, and is a homeomorphism if it is continuous and has a continuous inverse.) This means that $(\psi,(\Omega, g))$ is an extension of the closure space $\left(X, c_{\nu}\right)$. This extension is called the principal (or strict) extension of $\left(X, c_{\nu}\right)$ with trace system $\Omega$.

If in particular $(\Omega, g)$ is a topological space (this is the case if $\nu$ is a Lodato nearness), then it is easily seen that $g \alpha=\bigcap\left\{A^{*}: A^{*} \supset \alpha\right\}$ for all $\alpha \subset \Omega$, so that in this case $(\psi,(\Omega, g))$ is the classical principal (or strict) extension with trace system $\Omega$.

The closure space $\left(X^{\nu}, g\right)$ is $F$-linkage compact iff $\nu$ is a contigual nearness, it is linkage compact iff $\nu$ is a proximal nearness.

For more details, see Chattopadhyay, Njåstad and Thron [4].

4.2. Proposition. If $(X, \nu)$ is an $\Omega$-quasi-regular $T_{1}$ nearness space, then the principal extension $(\psi,(\Omega, g)$ ) is a regular closure space. (In particular, if $(X, \nu)$ is quasi-regular then $\left(\psi,\left(X^{\nu}, g\right)\right)$ is regular.)

Proof. Let $\alpha \subset \Omega, \bigodot_{0} \in \Omega$ and $\bigodot_{0} \notin g \alpha$. Then $\bigodot_{0} \notin\left(\psi^{-1}(\alpha)\right)^{*}$, and there exists an $A \subset X$ such that $A^{*} \supset \alpha-\psi(X)$ and $\bigodot_{0} \notin A^{*}$. Then $\psi^{-1}(\alpha) \cup A \notin \bigodot_{0}$. Since $(X, \nu)$ is $\Omega$-quasi-regular and $\bigodot_{0} \in \Omega$ it follows 
that there exist disjoint subsets $U, V$ of $X$ such that $U \in \sec \bigodot_{0}, V \in \sec \bigodot$ for all $e \in\left(\psi^{-1}(\alpha) \cup A\right)^{+}$. Set $\tilde{U}=\Omega-(X-U)^{+}, \tilde{V}=\Omega-$ $(X-V)^{+}$. Since $U \in \sec \bigodot_{0}$ it follows that $\bigodot_{0} \notin(X-U)^{*}$. Now $\Omega-$ $\tilde{U}=(X-U)^{+}$, and therefore $(\Omega-\tilde{U}) \cap \psi(X-U)=\psi(X-U)$ and $(\Omega-\tilde{U})-\psi(X-U)=(X-U)^{*}-\psi(X)$. Thus $\left(\psi^{-1}(\Omega-\tilde{U})\right)^{*}=$ $(X-U)^{*}$, and $(\Omega-\tilde{U})-\psi(X) \subset(X-U)^{*}$. It follows that $\bigodot_{0} \notin$ $g(\Omega-\tilde{U})$, which means that $\tilde{U}$ is a neighbourhood of $\bigodot_{0}$.

Let $\mathcal{C} \in \alpha$. Then either there is an $x \in X$ such that $\mathcal{C}=\psi(x)$, in which case $x \in \psi^{-1}(\alpha)$, or $\mathcal{C} \in \alpha-\psi(X)$, in which case $\mathcal{C} \in A^{*}$ so that $A \cup \psi^{-1}(\alpha) \in \mathcal{C}$. Thus in any case $\mathcal{C} \in\left(A \cup \psi^{-1}(\alpha)\right)^{+}$, hence $X-V \notin$

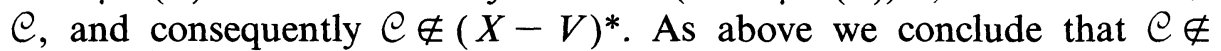
$g(\Omega-\tilde{V})$. Thus $\alpha \cap g(\Omega-\tilde{V})=\varnothing$, which means that $\tilde{V}$ is a neighbourhood of $\alpha$.

Now let $\circlearrowright \in \tilde{U}$. Then $\circlearrowright \notin(X-U)^{+}$.

First assume that $\mathcal{C}$ is principal. Then there is an $x \in U$ such that $\psi(x)=\bigodot$. Since $x \in U \subset X-V$ it follows that $\bigodot \notin \tilde{V}$.

Next assume that $C$ is non-principal. Then $(X-U) \notin \bigodot$ and hence $X-V \in \mathcal{C}$, since $(X-U) \cup(X-V)=X$. Consequently $e \in$ $(X-V)^{*}-\psi(X) \subset(X-V)^{+}$, so that $\bigodot \notin \tilde{V}$.

Therefore $\tilde{U}, \tilde{V}$ are disjoint neighbourhoods of $\bigodot_{0}$ and $\alpha$ respectively, and so $(\Omega, g)$ is regular.

4.3. Proposition. Let $(X, \nu)$ be a $T_{1}$ nearness space. If the principal extension $(\psi,(\Omega, g))$ is regular, then $(X, \nu)$ is $\Omega$-quasi-regular. (In particular, if $\left(\psi,\left(X^{\nu}, g\right)\right)$ is regular then $(X, \nu)$ is quasi-regular.)

Proof. Let $\bigodot_{0} \in \Omega, A \subset X$ and $A \notin \bigodot_{0}$. Then $\bigodot_{0} \notin A^{*}=g \psi(A)$. Since $g\left(A^{+}\right)=g \psi(A) \cup g\left(A^{*}-\psi(X)\right)=A^{*} \cup g\left(A^{*}-\psi(X)\right) \subset A^{*}$, it follows that $\bigodot_{0} \notin g\left(A^{+}\right)$. Since $(\Omega, g)$ is regular it follows that there are disjoint subsets $\beta, \delta$ of $\Omega$ such that $\bigodot_{0} \notin g(\Omega-\beta)$ and $A^{+} \cap g(\Omega-\delta)=$ $\varnothing$. Clearly $\psi^{-1}(\Omega-\beta) \notin \mathcal{C}_{0}$ and hence $\psi^{-1}(\beta) \in \sec \mathcal{C}_{0}$. Let $\mathcal{C} \in A^{+}$. Then $\bigodot \notin g(\Omega-\delta)$, hence $\psi^{-1}(\Omega-\delta) \notin \bigodot$ and consequently $\psi^{-1}(\delta) \in$

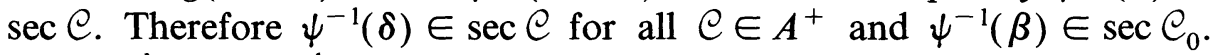
Also $\psi^{-1}(\beta) \cap \psi^{-1}(\delta)=\varnothing$, and so $(X, \nu)$ is $\Omega$-quasi-regular.

4.4. REMARK. Before proving the next result we note the following fact: A regular $T_{1}$ closure space is a $T_{2}$ space. This follows immediately from the definition of regularity, since in a $T_{1}$ space $(X, c)$ we have $c\{y\}=\{y\}$ for every point $y \in X$.

4.5. Proposition. Let $(X, \nu)$ be a $\Omega$-quasi-regular $T_{1}$ nearness space. Then each grill on $X$ is contained in at most one element of $\Omega$. 
Proof. By Proposition 3.8, $\left(X, c_{\nu}\right)$ is a regular closure space, hence also a $T_{2}$ space. Let $\mathcal{G}_{0}$ be a grill on $X$ and $\mathcal{G}_{0} \subset \bigodot_{1} \cap \mathcal{C}_{2}$, where $\mathcal{C}_{1}$ and $\bigodot_{2}$ belong to $\Omega$.

First assume that both $\mathcal{C}_{1}$ and $\bigodot_{2}$ are principal, e.g. $\mathcal{C}_{1}=\mathcal{G}\left(c_{\nu}, x\right)$, $\mathcal{C}_{2}=\mathcal{G}\left(c_{\nu}, y\right)$. Suppose $x \neq y$. Then there exist disjoint neighbourhoods $U, V$ of $x, y$ respectively. Clearly $U \in \sec \bigodot_{1} \subset \sec \mathcal{S}_{0}, V \in \sec \bigodot_{2} \subset$ $\sec \mathcal{G}_{0}$, and so $\varnothing=U \cap V \in \sec \mathcal{G}_{0}$, which is a contradiction. Thus $x=y$ and $e_{1}=e_{2}$.

Next assume that at least one of $\bigodot_{1}$ and $\bigodot_{2}$ are non-principal. Let e.g. $\bigodot_{2}$ be non-principal, and assume $\mathcal{C}_{1} \neq \mathcal{C}_{2}$. Let $A \in \mathcal{C}_{2}-\bigodot_{1}$. Since $A \notin \bigodot_{1}$ there exist disjoint subsets $U, V$ of $X$ such that $U \in \sec \bigodot_{1}$ and $V \in \sec \bigodot$ for all $\mathcal{C} \in A^{+}$. In particular $V \in \sec \mathcal{C}_{2}$. Thus $\varnothing=U \cap V \in \sec \mathcal{G}_{0}$, which again is a contradiction. Thus $e_{1}=e_{2}$ also in this case.

4.6. Remark. For every family $\mathbb{Q}$ let $\nu(\Theta)$ denote the family $\{B$ : $\{B\} \cup \mathbb{Q} \in \nu\}$. The nearness space $(X, \nu)$ is said to be separated if $\nu(\mathbb{Q})$ is in $\nu$ whenever $\mathbb{Q}$ and $\sec Q$ are in $\nu$. Every regular nearness space is separated. In a separated nearness space every clan is contained in exactly one cluster. See Herrlich [8, p. 87].

We may call the space $(X, \nu)$ quasi-separated if every clan is contained in at most one cluster. With this terminology Proposition 4.5 implies: A quasi-regular $T_{1}$ space is quasi-separated.

4.7. Definition. A subset $Y$ of a nearness space $(X, \nu)$ is said to be $\Omega$-clan-covered if each clan on the subspace $\left(Y, \nu_{Y}\right)$ of $(X, \nu)$ is contained in a cluster in $\Omega$. If $Y$ is $X^{\nu}$-clan-covered, we say that $Y$ is clan-covered. (We also say that $(X, \nu)$ is clan-covered if $X$ is clan-covered.)

4.8. Remark. According to Remark 4.6 every separated nearness space is clan-covered. In particular this is true for all regular nearness spaces, and a fortiori for all uniform nearness spaces, cf. §3.3. A quasi-regular nearness space need not be clan-covered, however, as the following example shows.

4.9. EXAMPLE. Let $(X, c)$ be the non-topological regular $T_{1}$ closure space of Example 3.2, and let $X_{n}, n=1,2, \ldots$, have the same meaning as in that example. Let $\mathscr{Q}_{i}$ be a non-principal ultrafilter on $X$ containing $X_{l}$, for all $i=1,2, \ldots$ Set $\mathcal{G}_{n}=\bigcup\left\{\mathscr{Q}_{i}: i \neq 2^{n}, 2^{n+1}, \ldots\right\}$ for all $n=1,2, \ldots$ Since $\mathcal{Q}_{i} \neq \mathcal{Q}_{J}$ for $i \neq j$ it follows that $\mathcal{G}_{n} \subset \mathcal{G}_{n+1}$ for all $n=1,2, \ldots$

We define a nearness $\sigma$ on $(X, c)$ by $\mathscr{Q} \in \sigma$ iff $\mathscr{Q} \subset \mathcal{G}(c, x)$ for some $x \in X$ or $\mathbb{C} \subset \mathcal{G}_{n}$ for some $n=1,2, \ldots$.

Clearly $(X, \sigma)$ is a Riesz nearness space and $c_{\sigma}=c$. Assume that there

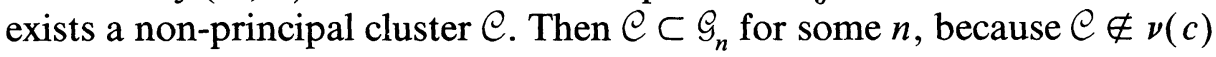


(recall the definition of $\nu(c)$ in $\$ 2.4$, cf. Example 3.13.). This is clearly impossible, since $\mathcal{G}_{n} \subset \mathcal{G}_{n+1}$. Thus $X^{\sigma}=\{\mathcal{G}(c, x): x \in X\}$.

Now every $\mathcal{G}_{n}$ is a clan, and $\mathcal{G}_{n}$ is not contained in a cluster. Hence $(X, \sigma)$ is not clan-covered.

Since $\left(X, c_{\sigma}\right)$ is regular and $X^{\sigma}=\Pi$, it follows from Proposition 3.10 that $(X, \sigma)$ is quasi-regular.

5. Extension of nearness-preserving maps. In this section we prove our main extension theorems. A map $f:(X, \nu) \rightarrow(Y, \mu)$ is said to be nearness-preserving if $f(\mathbb{Q}) \in \mu$, for every $Q \in \nu$.

5.1. THEOREM. Let $f$ be a nearness-preserving map from a $T_{1}$ nearness space $(X, \nu)$ into a $\Omega_{\mu}$-quasi-regular $T_{1}$ nearness space $(Y, \mu)$ and assume that $f(X)$ is $\Omega_{\mu}$-clan-covered. Then there exists a unique continuous extension $\bar{f}$ of $f$ from $\left(X^{\nu}, g_{\nu}\right)$ into $\left(\Omega_{\mu}, g_{\mu}\right)$.

$$
\begin{array}{ccc}
(X, \nu) & \stackrel{f}{\rightarrow} & (Y, \mu) \\
\psi_{\nu} \downarrow & & \downarrow \psi_{\mu} \\
\left(X^{\nu}, g_{\nu}\right) & \stackrel{\bar{f}}{\rightarrow} & \left(\Omega_{\mu}, g_{\mu}\right)
\end{array}
$$

Proof. We first describe the construction of the map $\bar{f}$. Let $\mathcal{C} \in X^{\nu}$. Then $\mathcal{H}=\{f(A): A \in \mathcal{C}\}$ is a grill on $f(X)$ and $\mathcal{K} \in \mu$ since $f$ is nearness-preserving. Thus $\mathcal{H}$ is a clan on the subspace $\left(f(X), \mu_{f(X)}\right)$ of $(Y, \mu)$. Then there is a $\mathcal{G}_{0}$ in $\Omega_{\mu}$ such that $\mathcal{H} \subset \mathcal{G}_{0}$, since $f(X)$ is $\Omega_{\mu}$-clancovered. Then also $\mathscr{K} \subset \mathcal{G}_{0}$, where $\mathscr{K}=\{B \subset Y: B \supset f(A)$ for some $A \in \mathcal{C}\}$. Since $\mathscr{K}$ is a grill on $Y$ and $(Y, \mu)$ is $\Omega_{\mu}$-quasi-regular it follows that $\mathcal{G}_{0}$ is the only element in $\Omega_{\mu}$ containing $\mathcal{H}$. We write $\tilde{f}(\mathcal{C})=\mathcal{G}_{0}$. This defines a map $\bar{f}$.

Clearly $\bar{f}\left(\psi_{\nu}(x)\right)=\bar{f}\left(\mathcal{G}\left(c_{\nu}, x\right)\right)=\mathcal{G}\left(c_{\mu}, f(x)\right)=\psi_{\mu}(f(x))$ for all $x \in X$. Thus $\bar{f}$ may be considered as an extension of $f$.

We shall prove that $f$ is continuous. Let $\alpha \subset X^{\nu}, \varrho \in X^{\nu}$ and $\bar{f}(\mathcal{C}) \notin$ $g_{\mu} \bar{f}(\alpha)$. We are going to show that $\bigodot \notin g_{\nu} \alpha$. Since $f\left(\psi_{\nu}^{-1}(\alpha)\right) \subset \psi_{\mu}^{-1}(\bar{f}(\alpha))$ we have

(i) $\psi_{\nu}^{-1}(\alpha) \notin e$.

This shows that $e \notin\left(\psi_{\nu}^{-1}(\alpha)\right)^{*}$.

Now since

$$
\begin{aligned}
\bar{f}(\mathcal{C}) \notin g_{\mu} \bar{f}(\alpha)=\left[\psi_{\mu}^{-1}(\bar{f}(\alpha))\right]^{*} \\
\\
\cup\left[\cap\left\{B^{*}: B \subset Y, B \supset \bar{f}(\alpha)-\psi_{\mu}(Y)\right\}\right],
\end{aligned}
$$


it follows that $\psi_{\mu}^{-1}(\bar{f}(\alpha)) \notin \bar{f}(\bigodot)$ and there exists a $D \subset Y$ such that $D^{*} \supset \bar{f}(\alpha)$ and $D \notin \bar{f}(\bigodot)$. Set $E=\psi_{\mu}^{-1}(\bar{f}(\alpha)) \cup D$. Then $E \notin \bar{f}(\bigodot)$. Since $(Y, \mu)$ is an $\Omega_{\mu}$-quasi-regular nearness space and $\bar{f}(\bigodot) \in \Omega_{\mu}$ it follows that there exist disjoint subsets $U, V$ of $Y$ such that $U \in \sec f(\bigodot)$ and $V \in \sec \mathscr{B}$ for all $\mathscr{B} \in E^{+}$.

Let $\mathcal{Q}$ be an arbitrary element of $\alpha$. Then $\bar{f}(\mathscr{Q}) \in \bar{f}(\alpha)$. First assume that $\bar{f}(\mathbb{Q})$ is principal. Then there is a a $y \in Y$ such that $\bar{f}(\mathbb{Q})=\psi_{\mu}(y)=$ $\mathcal{G}\left(c_{\mu}, y\right)$. Since $\psi_{\mu}(y) \in \bar{f}(\alpha)$ it follows that $y \in \psi_{\mu}^{-1}(\bar{f}(\alpha)) \subset E$. Thus $\bar{f}(\mathscr{Q}) \in E^{+}$. Next assume that $\bar{f}(\mathbb{Q})$ is non-principal. Then $f(\mathbb{Q}) \in f(\alpha)-$ $\psi_{\mu}(Y) \subset D^{*} \subset E^{*}$, and hence $\bar{f}(\mathbb{Q}) \in E^{+}$, since $f(\mathbb{Q}) \in \Omega_{\mu}-\psi_{\mu}(Y)$.

Thus in any case $\mathbb{Q} \in \alpha$ implies $\bar{f}(\mathbb{Q}) \in E^{+}$. It follows that $V \in$ $\sec \bar{f}(\mathbb{Q})$. Hence $X-f^{-1}(V) \notin \mathbb{Q}$, so that $\mathbb{Q} \in\left(f^{-1}(V)\right)^{\nu}$. This means

(ii) $\alpha \subset\left(f^{-1}(V)\right)^{\nu}$.

Also $U \in \sec \bar{f}(\mathcal{C})$ so that $Y-U \notin \bar{f}(\mathcal{C})$, and hence $V \notin \bar{f}(\mathcal{C})$. Then $f^{-1}(V) \notin \mathcal{C}$ and consequently

(iii) $巳 \notin\left(f^{-1}(V)\right)^{\nu}$.

This shows that there exists a set $A \subset X$ such that $\alpha \subset A^{\nu}, \bigodot \notin A^{\nu}$.

Now $g_{\nu} \alpha=\left[\psi_{\nu}^{-1}(\alpha)\right]^{\nu} \cup\left[\cap\left\{A^{\nu}: A \subset X, A^{\nu} \supset \alpha\right\}\right]$. By the foregoing

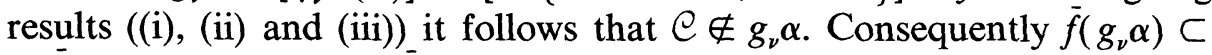
$g_{\mu} \bar{f}(\alpha)$. This shows that $\bar{f}$ is continuous.

Since $\left(\Omega_{\mu}, g_{\mu}\right)$ is a regular $T_{1}$ space by Proposition 4.2 it follows that $\left(\Omega_{\mu}, g_{\mu}\right)$ is a $T_{2}$ space. Furthermore $\psi_{\nu}(X)$ is dense in $\left(X^{\nu}, g_{\nu}\right)$, and consequently the continuous extension $f$ is unique.

5.2. THEOREM. Let $f$ be a nearness-preserving map from a $T_{1}$ nearness space $(X, \nu)$ into a quasi-regular clan-covered $T_{1}$ nearness space $(Y, \mu)$. Then there exists a unique continuous extension $f$ of $f$ from $\left(X^{\nu}, g_{\nu}\right)$ into $\left(Y^{\mu}, g_{\mu}\right)$.

We have stated this important special case of Theorem 5.1 as a separate theorem. The proof is immediate.

Concerning the following result, cf. Herrlich [8, p. 95-96].

5.3. COROLlaRY. Let $f$ be a nearness-preserving map from a $T_{1}$ Lodato nearness space $(X, \nu)$ into a regular $T_{1}$ Lodato nearness space $(Y, \mu)$. Then there exists a unique continuous extension $\bar{f}$ of $f$ from $\left(X^{\nu}, g_{\nu}\right)$ into $\left(Y^{\mu}, g_{\mu}\right)$.

Proof. Since $(Y, \mu)$ is a regular nearness space it is also quasi-regular and clan-covered (cf. Remark 4.8), and so the result follows immediately from Theorem 5.2. 
5.4. Definition. A subspace $\left(Y, \eta_{2}\right)$ of a nearness space $\left(\lambda Y, \eta_{1}\right)$ is said to be relatively clan complete in $\left(\lambda Y, \eta_{1}\right)$ if for every $\eta_{2}$-clan $e$ in $\left(Y, \eta_{2}\right)$ there exists an $x \in \lambda Y$ such that $\{\{x\}\} \cup \bigodot \in \eta_{1}$.

This definition was introduced by Naimpally [11, p. 249]. From Theorem 5.1 we can now easily obtain the following extension theorem of Naimpally [11, p. 251]. (For the meaning of $\nu(k)$, see §2.4.)

5.5. THEOREM. Let $(X, c)$ be a dense subspace of a topological $T_{1}$ space $(\alpha X, k)$ and let $\nu$ be the restriction to $X$ of the nearness $\nu(k)$ on $\alpha X$. Let $(\lambda Y, \mu)$ be an $R$-nearness space such that $\left(\lambda Y, c_{\mu}\right)$ is a $T_{1}$ space, and let $\left(Y, \mu_{0}\right)$ be a subspace of $(\lambda Y, \mu)$ which is relatively clan complete in $(\lambda Y, \mu)$. Then every nearness-preserving map $f:(X, \nu) \rightarrow\left(Y, \mu_{0}\right)$ has a unique continuous extension $F:(\alpha X, k) \rightarrow\left(\lambda Y, c_{\mu}\right)$.

Proof. Let $\tau(x)=\{A \subset X: x \in k A\}$ for all $x \in \alpha X$. Then $X^{\nu}=$ $\{\tau(x): x \in \alpha X\}$ is the set of $\nu$-clusters. We first show that $\tau:(\alpha X, k) \rightarrow$ $\left(X^{\nu}, g_{\nu}\right.$ ) is continuous. Let $x \in \alpha X, C \subset \alpha X$, and assume $\tau(x) \notin g_{\nu} \tau(C)$. Then $\tau(x) \notin D^{\nu}$, where $D=\psi_{\nu}^{-1}(\tau(C))=C \cap X$, and there exists a set $E \subset X$ such that $E^{\nu} \supset \tau(C)-\psi_{\nu}(X)$ and $\tau(x) \notin E^{\nu}$. Since $D \notin \tau(x)$ and $E \notin \tau(x)$ we also have $D \cup E \notin \tau(x)$. This means by definition of the map $\tau$ that $x \notin k(D \cup E)$. Clearly $k D \supset C \cap X$, and we also have $k E \supset C-X$. (For let $y \in C-x$. Then $\tau(y) \in \tau(C)-\psi_{\nu}(X) \subset E^{\nu}$, hence $E \in \tau(y)$ which means $y \in k E$. Thus $C-X \subset k E$.) It follows that $k(D \cup E) \supset(C \cap X) \cup(C-X)=C$, and therefore also $k(D \cup E) \supset$ $k C$, which implies $x \notin k C$. It follows that $\tau$ is continuous.

Since $Y$ is relatively clan complete in $(\lambda Y, \mu)$ and $f(X) \subset Y$, we infer that $f(X)$ is $\Omega_{\mu}$-clan-covered, where $\Omega_{\mu}=\left\{\mathcal{G}\left(c_{\mu}, y\right): y \in \lambda Y\right\}$. Since $(\lambda Y, \mu)$ is an $R$-nearness space it follows that $\left(\lambda Y, c_{\mu}\right)$ is a regular topological space (see Section 3.4). Hence $(\lambda Y, \mu)$ is $\Omega_{\mu}$-quasi-regular, by Proposition 3.10. Furthermore $\left(\lambda Y, c_{\mu}\right)$ is homeomorphic to $\left(\Omega_{\mu}, g_{\mu}\right)$. Therefore by Theorem 5.1 there is a continuous extension $\bar{f}:\left(X^{\nu}, g_{\nu}\right) \rightarrow$ $\left(\lambda Y, c_{\mu}\right)$ of $f$, in the sense that the following diagram commutes.

$$
\begin{array}{ccc}
(X, \nu) & \stackrel{f}{\rightarrow} & \left(Y, \mu_{0}\right) \\
\psi_{\nu} \downarrow & & \downarrow i_{Y} \\
\left(X^{\nu}, g_{\nu}\right) & \stackrel{\bar{f}}{\rightarrow} & \left(\lambda Y, c_{\mu}\right)
\end{array}
$$

Also $\tau$ is a continuous map on $(\alpha X, k)$, so that the following diagram commutes.

$$
\begin{array}{ccc} 
& \left(X, c_{\nu}\right) \\
& & \\
& i_{X} \swarrow & \searrow \psi_{\nu} \\
\rightarrow & \left(X^{\nu}, g_{\nu}\right)
\end{array}
$$


Thus $F=f \circ \tau$ is a continuous map such that the following diagram commutes.

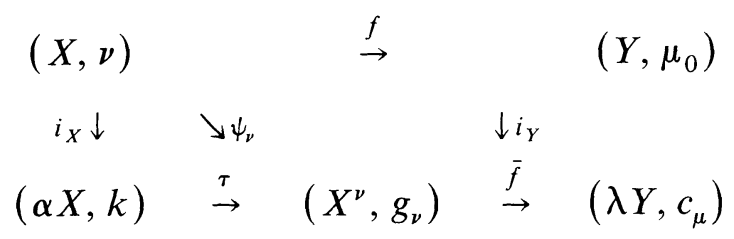

Since $\left(\lambda Y, c_{\mu}\right)$ is a $T_{2}$ space and $X$ is dense in $(\alpha X, k)$ it follows that the continuous extension is unique.

5.6. REMARK. Earlier known extension results related to our main theorems have been proved only for Lodato nearness spaces. We shall show that there exist non-Lodato nearness spaces where the conditions of our Theorem 5.2 are satisfied. Thus our results cover situations where earlier known results do not apply.

Let $(X, c)$ be the closure space of Example 3.2, and let $\nu$ be the nearness $\nu(c)$ on $X$ (cf. §2.4). In Example 3.13 we showed that $(X, \nu)$ is a quasi-regular $T_{1}$ nearness space such that the induced closure space $\left(X, c_{\nu}\right)$ equals $(X, c)$ and is therefore non-topological. The space $(X, \nu)$ is evidently clan-covered, since every clan is contained in some principal cluster. In view of Theorem 5.2 every nearness-preserving map from a $T_{1}$ nearness space $(Z, \zeta)$ into $(X, \nu)$ has a unique extension to a continuous $\operatorname{map} \bar{f}:\left(Z^{\zeta}, g_{\zeta}\right) \rightarrow\left(X, c_{\nu}\right)$. (Here $X^{\nu}=\Pi$, and $\left(\Pi, g_{\nu}\right)$ is homeomorphic to $\left(X, c_{\nu}\right)$.)

Let $Y$ and $J$ have the same meaning as in Example 3.2. Let $\rho$ be the restriction of the nearness $\nu$ to $Y \cup J=X-\{\omega\}$. The space $(Y \cup J, \rho)$ is then quasi-regular and clan-covered. Again the space is not a Lodato space, even though the closure space $\left(Y \cup J, c_{\rho}\right)$ is topological. Again it follows from Theorem 5.2 that every nearness-preserving map from a $T_{1}$ nearness space $(Z, \zeta)$ into $(Y \cup J, \rho)$ has a unique extension to a continuous map $\bar{f}:\left(Z^{\zeta}, g_{\zeta}\right) \rightarrow\left(X, c_{\nu}\right)$. (The principal extension $\left((J \cup Y)^{\rho}, g_{\rho}\right)$ is easily seen to be homeomorphic to $(X, \nu)$.)

6. Extension of continuous maps. We shall now discuss continuous extension of continuous maps to closure space extensions which are linkage compact or $F$-linkage compact (cf. \$2.2). In order to be able to obtain such extensions we need to introduce conditions on closure spaces more stringent than regularity. These conditions are formulated in terms of the nearnesses $\xi(c)$ and $\pi(c)$, but are clearly statements about the closure space $(X, c)$. (For the definition of the nearnesses $\xi(c)$ and $\pi(c)$ see Section 2.4.) 
6.1. Definition. A $T_{1}$ closure space $(X, c)$ is called $C$-regular if the contigual nearness space $(X, \xi(c))$ is quasi-regular.

6.2. Proposition. A $T_{1}$ F-linkage compact closure space is $C$-regular iff it is regular.

Proof. If $(X, c)$ is $C$-regular then $(X, \xi(c))$ is quasi-regular and $c_{\xi(c)}=c$. Hence by Proposition 3.8, $(X, c)$ is regular.

Next suppose that $(X, c)$ is regular. Let $\mathcal{C}$ be a $\xi(c)$-cluster. Then $\{c A$ : $A \in \mathcal{C}\}$ has the finite intersection property. Since $(X, c)$ is $F$-linkage compact there is an $x \in X$ such that $\mathcal{C} \subset \mathcal{G}(c, x)$, and so $\mathcal{C}=\mathcal{G}(x, c)$ since $\mathcal{G}(c, x)$ is a $\xi(c)$-cluster. Hence $\{\mathcal{G}(c, x): x \in X\}$ is the set of all $\xi(c)$-clusters. By Proposition 3.10 it now follows that $(X, \xi(c))$ is quasi-regular, which means that $(X, c)$ is $C$-regular.

6.3. Proposition. $A T_{1}$ closure space $(X, c)$ is $C$-regular iff the F-linkage compact principal extension $\left(\psi_{\xi(c)},\left(X^{\xi(c)}, g_{\xi(c)}\right)\right)$ is C-regular.

Proof. Let $(X, c)$ be $C$-regular. Then $(X, \xi(c))$ is quasi-regular. Hence by Proposition $4.2,\left(X^{\xi(c)}, g_{\xi(c)}\right)$ is regular. Since $\left(X^{\xi(c)}, g_{\xi(c)}\right)$ is an $F$-linkage compact regular $T_{1}$ space it follows by Proposition 6.2 that $\left(X^{\xi(c)}, g_{\xi(c)}\right)$ is $C$-regular.

Next assume that $\left(X^{\xi(c)}, g_{\xi(c)}\right)$ is $C$-regular. Then it is also regular by Proposition 6.2. Hence by Proposition 4.3, $(X, \xi(c))$ is quasi-regular, which means that $(X, c)$ is $C$-regular.

6.4. Proposition. Let $(X, c)$ and $(Y, d)$ be $T_{1}$ closure spaces. A map $f:(X, c) \rightarrow(Y, d)$ is continuous iff $f:(X, \xi(c)) \rightarrow(Y, \xi(d))$ is a nearnesspreserving map.

Proof. Let $f:(X, \xi(c)) \rightarrow(Y, \xi(d))$ be a nearness-preserving map. Assume $x \in c A$. Then $\{\{x\}, A\} \in \xi(c)$ and hence $\{\{f(x)\}, f(A)\} \in \xi(d)$, and consequently $f(x) \in d f(A)$. Thus $f(c A) \subset d f(A)$, which means that $f$ is continuous from $(X, c)$ to $(Y, d)$.

Next assume that $f:(X, c) \rightarrow(Y, d)$ is continuous. Let $\mathbb{Q} \in \xi(c)$, and let $\left\{A_{1}, A_{2}, \ldots, A_{n}\right\}$ be an arbitrary finite subfamily of $\mathbb{Q}$. Then there is an $x \in \cap_{i=1}^{n} c A_{i}$. Since $f$ is continuous, $f(x) \in \cap_{i=1}^{n} d f\left(A_{i}\right)$. Since $\left\{A_{1}, A_{2}, \ldots, A_{n}\right\}$ is an arbitrary finite subfamily of $\mathbb{Q}$ it follows that $f(\mathbb{Q}) \in \xi(d)$. Thus the map $f:(X, \xi(c)) \rightarrow(Y, \xi(d))$ is nearness-preserving. 
6.5. TheOREM. Let $(X, c)$ be a $T_{1}$ closure space and let $(Y, d)$ be a regular F-linkage compact $T_{1}$ closure space. Let $f:(X, c) \rightarrow(Y, d)$ be a continuous map. Then there exists a unique continuous extension $\bar{f}$ of from $\left(X^{\xi(c)}, g_{\xi(c)}\right)$ into $(Y, d)$.

Proof. By Proposition 6.4, $f:(X, \xi(c)) \rightarrow(Y, \xi(d))$ is a nearness-preserving map. By Proposition 6.2, $(Y, d)$ is $C$-regular. Therefore $(Y, \xi(d))$ is quasi-regular. Since $(Y, d)$ is $F$-linkage compact, $Y^{\xi(d)}=\{\mathcal{G}(d, y)$ : $y \in Y\}$. Clearly $(Y, \xi(d))$ is clan-covered. So by Theorem 5.2 , there is a unique continuous extension $f:\left(X^{\xi(c)}, g_{\xi(c)}\right) \rightarrow\left(Y^{\xi(d)}, g_{\xi(d)}\right)$ of $f$. Finally note that $(Y, d)$ is homeomorphic to $\left(Y^{\xi(d)}, g_{\xi(d)}\right)$. Hence there is a unique continuous extension $\bar{f}:\left(X^{\xi(c)}, g_{\xi(c)}\right) \rightarrow(Y, d)$ of $f$, since $(Y, d)$ is a $T_{2}$ space and $\psi_{\xi(c)}(X)$ is dense in $\left(X^{\xi(c)}, g_{\xi(c)}\right)$.

6.6. Let $C$-REG denote the category of $C$-regular $T_{1}$ closure spaces and continuous maps. (For categorical background, see e.g. Herrlich and Strecker [7].) Let FLC-REG denote the category of regular $F$-linkage compact $T_{1}$ closure spaces and continuous maps. In view of Proposition 6.2 we may say that FLC-REG is a full subcategory of $C$-REG. Note that if $(X, c)$ is a $C$-regular $T_{1}$ closure space, then by Proposition 6.3, $\left(\psi_{\xi(c)},\left(X^{\xi(c)}, g_{\xi(c)}\right)\right)$ is an $F$-linkage compact $C$-regular extension of $(X, c)$. In view of Theorem 6.5 we then have the following result:

6.7. THEOREM. FLC-REG is epi-reflective in $C-R E G$. The reflection of an object $(X, c)$ of $C-R E G$ in FLC-REG is $\left(\psi_{\xi(c)},\left(X^{\xi(c)}, g_{\xi(c)}\right)\right)$.

6.8. Definition. A $T_{1}$ closure space $(X, c)$ is called $P$-regular if the proximal nearness space $(X, \pi(c))$ is quasi-regular.

By arguments analogous to the preceding ones we get the following results:

6.9. Proposition. A $T_{1}$ linkage compact closure space is $P$-regular iff it is regular.

6.10. Proposition. $A T_{1}$ closure space $(X, c)$ is $P$-regular iff the linkage compact principal extension $\left(\psi_{\pi(c)},\left(X^{\pi(c)}, g_{\pi(c)}\right)\right)$ is $P$-regular.

6.11. Proposition. Let $(X, c)$ and $(Y, d)$ be $T_{1}$ closure spaces. A map $f:(X, c) \rightarrow(Y, d)$ is continuous iff $f:(X, \pi(c)) \rightarrow(Y, \pi(d))$ is a nearnesspreserving map. 
6.12. Theorem. Let $(X, c)$ be a $T_{1}$ closure space and let $(Y, d)$ be a regular linkage compact $T_{1}$ closure space. Let $f:(X, c) \rightarrow(Y, d)$ be a continuous map. Then there exists a unique continuous extension $\bar{f}$ of $f$ from $\left(X^{\pi(c)}, g_{\pi(c)}\right)$ into $(Y, d)$.

6.13. Let $P$-REG denote the category of $P$-regular $T_{1}$ closure spaces and continuous maps. Let $L C$-REG denote the category of regular linkage compact $T_{1}$ closure spaces and continuous maps. By Proposition 6.9, it follows that $L C$-REG is a full subcategory of $P$-REG. If $(X, c)$ is a $P$-regular $T_{1}$ closure space then by Proposition 6.10, $\left(\psi_{\pi(c)},\left(X^{\pi(c)}, g_{\pi(c)}\right)\right)$ is a linkage compact $P$-regular extension of $(X, c)$. In view of Theorem 6.12 we then have the following result:

6.14. THEOREM. LC-REG is epi-reflective in P-REG. The reflection of an object $(X, c)$ of P-REG in LC-REG is $\left(\psi_{\pi(c)},\left(X^{\pi(c)}, g_{\pi(c)}\right)\right)$.

6.15. Remark. For a topological $T_{1}$ space $(X, c)$ the extension $\left(X^{\xi(c)}, g_{\xi(c)}\right)$ is equivalent to the classical Wallman compactification. If $(X, c)$ is also $C$-regular, then the extension $\left(X^{\xi(c)}, g_{\xi(c)}\right)$ is a $T_{2}$ compactification, and so by Theorem 6.5 it is equivalent to the Stone-Čech compactification. It follows that $(X, c)$ in this case is a normal space.

\section{REFERENCES}

1. H. L. Bentley, Nearness spaces and extensions of topological spaces, Studies in Topology, N. Stavrakas and K. Allen, Ed., Academic Press, New York, 1975, 47-66.

2. H. L. Bentley and H. Herrlich, Extensions of Topological Spaces, Proceedings of the Memphis State University Conference in Topology, Stanley P. Franklin and Barbara V. Smith Thomas, Ed., Lecture Notes in Pure and Applied Mathematics, Marcel Dekker, New York, 1976, 129-184.

3. K. C. Chattopadhyay and W. J. Thron, Extensions of closure spaces, Canad. J. Math., 29 (1977), 1277-1286.

4. K. C. Chattopadhyay, Olav Njåstad and W. J. Thron, Nearness structures and extensions of closure spaces, submitted.

5. E. Čech, Topological Spaces, rev. ed. (Publ. House Czech. Acad. Sc. Prague, English transl. Wiley, New York, 1966).

6. D. Harris, Regular-closed spaces and proximities, Pacific J. Math., 34 (1970), 675-685.

7. H. Herrlich and C. E. Strecker, Category Theory, Allyn and Bacon, Boston, 1973.

8. H. Herrlich, Topological Structures, in: Topological structures, P. C. Baayen, Ed., Math. Centre Tracts 52, Amsterdam (1974), 59-122.

9. M. Katětov, On continuity structures and spaces of mappings, Comm. Math. Univ. Carolinae, 6 (1965), 257-278.

10. K., Morita, On the simple extension of a space with respect to a uniformity I-IV, Proc. Japan Acad., 27 (1951), 65-72, 130-137, 166-171, 632-636.

11. S. A. Naimpally, Reflective functors via nearness, Fund. Math., 85 (1974), 245-255.

12. Ellen E. Reed, Nearnesses, proximities and $T_{1}$-compactificatons. Trans. Amer. Math.

Soc., 236 (1978), 193-207. 
13. A. K. Steiner and E. F. Steiner, On semiuniformities, Fund. Math., 83 (1973), 47-58.

14. W. J. Thron, Proximity structures and grills, Math. Ann., 206 (1973), 35-62.

Received May 8, 1981 and in revised form March 9, 1982.

BURDWAN UNIVERSITY

BURDWAN

WEST BENGAL, INDIA

AND

UNIVERSITY OF TRONDHEIM-NTH

TRONDHEIM, NORWAY 



\section{PACIFIC JOURNAL OF MATHEMATICS}

EDITORS

Donald BABBITT (Managing Editor)

University of California

Los Angeles, CA 90024

Hugo Rossi

University of Utah

Salt Lake City, UT 84112

C. C. Moore and Arthur Ogus

University of California

Berkeley, CA 94720
J. DugunduI

Department of Mathematics

University of Southern California

Los Angeles, CA 90089-1113

R. FinN and H. SAmelson

Stanford University

Stanford, CA 94305

\section{ASSOCIATE EDITORS}
R. ARENS
E. F. BECKENBACH
B. H. NEUMANN
F. WolF
K. YoshidA (1906-1982)

\section{SUPPORTING INSTITUTIONS}

UNIVERSITY OF ARIZONA

UNIVERSITY OF BRITISH COLUMBIA

CALIFORNIA INSTITUTE OF TECHNOLOGY

UNIVERSITY OF CALIFORNIA

MONTANA STATE UNIVERSITY

UNIVERSITY OF NEVADA, RENO

NEW MEXICO STATE UNIVERSITY

OREGON STATE UNIVERSITY
UNIVERSITY OF OREGON

UNIVERSITY OF SOUTHERN CALIFORNIA

STANFORD UNIVERSITY

UNIVERSITY OF HAWAII

UNIVERSITY OF TOKYO

UNIVERSITY OF UTAH

WASHINGTON STATE UNIVERSITY

UNIVERSITY OF WASHINGTON 


\section{Pacific Journal of Mathematics}

Vol. 105, No. 1 September, 1983

Kenneth F. Andersen, On the transformation of Fourier coefficients of

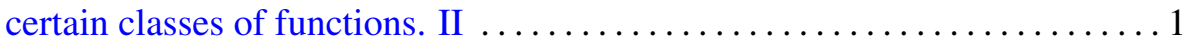

Gavin Brown, Irving Leonard Glicksberg and Edwin Hewitt, Indicator functions with large Fourier transforms $\ldots \ldots \ldots \ldots \ldots \ldots \ldots \ldots \ldots \ldots$

Shih-Sen Chang, Some random fixed point theorems for continuous random

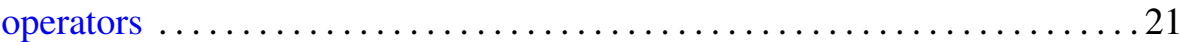

K. C. Chattopadhyay and Olav Njstad, Quasiregular nearness spaces and

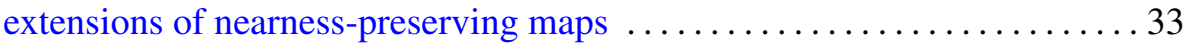

Thomas W. Cusick, The two-dimensional Diophantine approximation

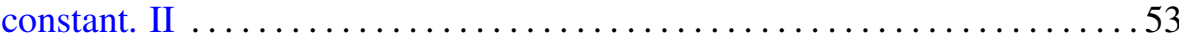

Eric Karel van Douwen and Jan van Mill, Spaces without remote points . . .669 Hector O. Fattorini, Convergence and approximation theorems for

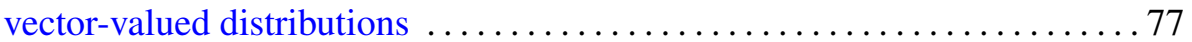

John J. F. Fournier and Louis Pigno, Analytic and arithmetic properties of

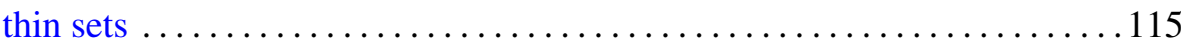

David Goss, On a new type of $L$-function for algebraic curves over finite

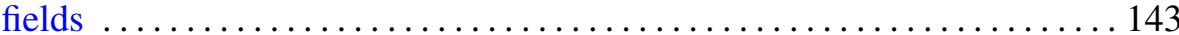

Douglas Austin Hensley, Lattice vertex polytopes with interior lattice

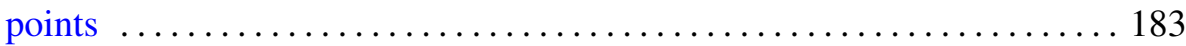

J. K. Kohli, Monotone extensions of mappings and their applications ...... 193

John C. Morgan, II, On equivalent category bases . . . . . . . . . . . . 207

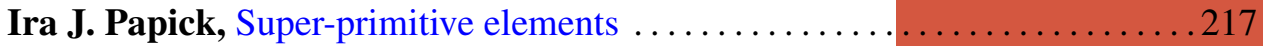

José Luis Rubio de Francia and José Luis Torrea, Vector extensions of

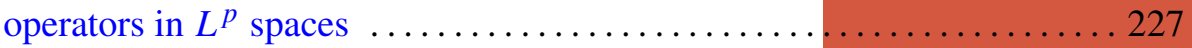

Mark Phillip Thomas, Closed ideals of $l^{1}\left(\omega_{n}\right)$ when $\left\{\omega_{n}\right\}$ is star-shaped $\ldots 237$ 